\section{Neural correlates of effort-dependent and effort-independent cognitive fatigue components in patients with multiple sclerosis}

\author{
Stefan Spiteri, Thomas Hassa, Dolores Claros-Salinas, Christian Dettmers and Mircea Ariel \\ Schoenfeld
}

\section{Abstract}

Background: Among patients with multiple sclerosis (MS), fatigue is the most commonly reported symptom. It can be subdivided into an effort-dependent (fatigability) and an effort-independent component (trait-fatigue).

Objective: The objective was to disentangle activity changes associated with effort-independent "traitfatigue" from those associated with effort-dependent fatigability in MS patients.

Methods: This study employed behavioral measures and functional magnetic imaging to investigate neural changes in MS patients associated with fatigue. A total of $40 \mathrm{MS}$ patients and 22 age-matched healthy controls performed in a fatigue-inducing $N$-back task. Effort-independent fatigue was assessed using the Fatigue Scale of Motor and Cognition (FSMC) questionnaire.

Results: Effort-independent fatigue was observed to be reflected by activity increases in fronto-striatalsubcortical networks primarily involved in the maintenance of homeostatic processes and in motor and cognitive control. Effort-dependent fatigue (fatigability) leads to activity decreases in attention-related cortical and subcortical networks.

Conclusion: These results indicate that effort-independent (fatigue) and effort-dependent fatigue (fatigability) in MS patients have functionally related but fundamentally different neural correlates. Fatigue in MS as a general phenomenon is reflected by complex interactions of activity increases in control networks (effort-independent component) and activity reductions in executive networks (effort-dependent component) of brain areas.

Keywords: Multiple sclerosis, fatigue, fatigability, state/trait-fatigue, attention, fMRI

Date received: 29 March 2017; revised: 5 October 2017; accepted: 18 October 2017

\section{Introduction}

One of the most prevalent and most common symptoms in multiple sclerosis (MS) is fatigue ${ }^{1}$ affecting approximately $70 \%-90 \%$ of patients as one of the most disabling symptoms in MS. ${ }^{2,3}$ Chaudhuri and Behan ${ }^{4}$ distinguish between physical fatigue, as the inability to sustain a specified work rate during exercise, and cognitive fatigue, as a failure of physical and mental tasks that require self-motivation and internal cues in the absence of motor weakness. They suggest that the cause of cognitive fatigue can be found in a failure of the non-motor functions of the basal ganglia (BG). Both forms of fatigue can be separated into an effort-independent general subjective sensation of fatigue, and fatigability as an effort-dependent change in performance. ${ }^{5}$

Functional imaging work on different fatigue types ${ }^{6}$ a correlation between fatigue and brain atrophy or lesion load. ${ }^{7}$ Others described a relationship with white matter lesions in cortical and subcortical areas ${ }^{8,9}$ or no association between fatigue and lesion load, lesion distribution, lesion location, or brain atrophy at all. ${ }^{8,10}$

Recent work ${ }^{4}$ proposed a close link between cognitive fatigue in MS and non-motor dysfunctions of the BG due to a neurotransmitter imbalance. Consequently, provided at least in part conflicting results. Some found
Multiple Sclerosis Journal 2019, Vol. 25(2) 256-266 DOI: $10.1177 /$ 1352458517743090

(C) The Author(s), 2017. Article reuse guidelines: sagepub.com/journalspermissions
Correspondence to: S Spiteri

Neurological Rehabilitation Center Kliniken Schmieder, Zum Tafelholz 8, 78476

Allensbach, Germany. s.spiteri@klinikenschmieder.de

Stefan Spiteri Thomas Hassa Lurija Institute for Rehabilitation and Health Sciences, Allensbach, Germany/Neurological Rehabilitation Center Kliniken Schmieder, Allensbach, Germany

Dolores Claros-Salinas Lurija Institute for Rehabilitation and Health Sciences, Allensbach, Germany/Neurological Rehabilitation Center Kliniken Schmieder, Konstanz, Germany Christian Dettmers Lurija Institute for Rehabilitation and Health Sciences, Allensbach, Germany/Neurological Rehabilitation Center Kliniken Schmieder, Konstanz, Germany/ Department of Psychology, University of Konstanz, Konstanz, Germany Mircea Ariel Schoenfeld Lurija Institute for Rehabilitation and Health Sciences, Allensbach, Germany/Neurological Rehabilitation Center Kliniken Schmieder, Heidelberg, Germany/ Department of Neurology, Otto-von-GuerickeUniversity, Magdeburg, Germany/Leibniz-Institute for Neurobiology, Magdeburg, Germany 
fatigue was suggested to arise from a dysfunction of the cortico-striatal network between prefrontal cortical (PFC) areas and the BG. ${ }^{11}$ This network also comprises the anterior cingulate cortex (ACC) that is also involved in the control of attention as part of a more distributed network. ${ }^{12,13}$ Similar to the abovementioned taxonomy of fatigue and fatigability, Genova et al. ${ }^{10}$ proposed to divide fatigue into two components: "trait" fatigue, referring to the experience of fatigue across a long period of time, which is not likely to change significantly over time, and "state" fatigue, which is referred to as a transient dynamic condition fluctuating based on internal and external factors. The authors employed functional magnetic resonance imaging (fMRI) in conjunction with a cognitive fatigue-inducing task-switching paradigm and structural measures in MS patients to investigate "state" and "trait" fatigue, respectively. They found higher activity in the caudate nucleus when compared to healthy controls, but no differences in gray matter (GM) volume or lesion load. While there were no differences in task performance (accuracy and reaction times (RTs)) between patients and controls, the subjective ratings of fatigue as well as activity in associated neural networks were higher in patients. ${ }^{10}$ The authors interpreted this discrepancy as supporting the notion that neuropsychological tests do not necessarily offer the most sensitive measure for fatigue. Diffusion tensor imaging (DTI) in conjunction with fatigue scores ${ }^{3}$ revealed a reduced fractional anisotropy (FA) within the anterior internal capsule that is connected to the caudate and thalamus supporting the idea of an important role of the striatal-thalamic-frontal system in fatigue. ${ }^{10}$

Recent studies provided strong evidence that the performance in alertness and vigilance tasks is more correlated with the subjective feeling of fatigue in MS patients, than other cognitive domains. This was observed following a period of roughly 15 minutes performing a monotonous task, arguing for a depletion of attentional resources. ${ }^{14}$ The neuroanatomical correlates include areas of the brainstem, midbrain, and thalamus, as well as fronto-parietal regions and the ACC. So far, functional imaging was mostly focused on motor fatigue and only few studies investigated cognitive fatigue. ${ }^{14}$ The aim of this study was to investigate the neural correlates of effort-dependent and effort-independent fatigue in MS patients and healthy controls. Effort-independent components correspond to previous definitions of "trait" fatigue, while the effort-dependent components correspond to "state" fatigue. Effort-independent fatigue correlates were assessed using the difference of activity in patients at the beginning of a cognitive task $(N$-back) that correlated with subjective evaluations of cognitive fatigue. It was assumed that the effects of effortdependent fatigue would be minimal at the beginning of the task. Effort-dependent fatigue correlates were assessed as the difference between the end and the beginning of the task. Behavioral and fatigue measures were performed before and after an $\mathrm{N}$-back task. Neural correlates of effort-independent fatigue were expected to be reflected by alterations of hemodynamic activity in cortical and subcortical network subserving cognitive and motor control. In contrast, effort-dependent fatigue correlates were expected in cortical executive systems, such as the associative visual cortex, which is more involved in the processing of the task itself.

\section{Materials and methods}

\section{Participants}

A total of 40 right-handed patients (31 female, aged 27-61) with definitive MS and normal or corrected-tonormal vision (acuity $>0.4$ ) participated in the study. We included patients with MS irrespective of their fatigue in order to be able to perform correlative analyses. Gait and manual functions were assessed by means of the clinical reports. Exclusion criteria for the patient group were psychiatric or neurological diseases other than MS or any contraindications for the magnetic resonance imaging (MRI). Age- and gender-matched control group of 22 healthy controls (15 female, aged 21-56) was recruited. The characteristics of both groups are shown in Table 1. All participants gave informed written consent and the study was approved by the local ethics committee (University of Konstanz, Germany).

\section{Behavioral measurements}

Patients completed the Fatigue Scale of Motor and Cognition (FSMC) ${ }^{15}$ questionnaire, as well as the Becks Depression Inventory (BDI $)^{16}$ on the day of the experiment. Participants performed in the alertness task from the Test-battery of Attentional Performance (TAP),${ }^{17}$ a simple visual reaction task shown to be sensitive to cognitive fatigue ${ }^{18-20}$ before and after the MRI measurement.

MRI. A 3T Siemens Skyra (Siemens, Erlangen) MR scanner with a standard 32-channel head coil was used for imaging:

Structural MRI. A T1-whole-brain-scan: 176 slices, voxel resolution: $1 \mathrm{~mm} \times 1 \mathrm{~mm} \times 1 \mathrm{~mm}$, repetition time $(\mathrm{TR})=2.7$ seconds, echo time $(\mathrm{TE})=7.21 \mathrm{~ms}$, no gap. 
Table 1. Descriptive statistics for the patient and control cohorts.

\begin{tabular}{|c|c|c|}
\hline & Patients $(N=40)$ & Controls $(N=22)$ \\
\hline Gender (M/F) & $9 / 31(22.5 \% / 77.5 \%)$ & $7 / 15(32 \% / 68 \%)$ \\
\hline \multicolumn{3}{|l|}{ Age in years } \\
\hline Mean (SD) & $46.75( \pm 7.53)$ & $41.73( \pm 12.52)$ \\
\hline Range & $27-61$ & $21-56$ \\
\hline Formal education in years & $11.5( \pm 1.7)$ & $12.1( \pm 1.4)$ \\
\hline EDSS & & N/A \\
\hline Overall mean (SD) & $3.5( \pm 1.5)$ & \\
\hline $0-1.5$ & $2(5 \%)$ & \\
\hline $2-4$ & $31(77.5 \%)$ & \\
\hline $4.5-6$ & $4(10 \%)$ & \\
\hline$\geq 6.5$ & $3(7.5 \%)$ & \\
\hline \multicolumn{3}{|l|}{ Disease duration in years } \\
\hline Mean (SD) & $14.1( \pm 8.8)$ & N/A \\
\hline $1-5$ years & $10(25 \%)$ & \\
\hline $6-10$ years & $14(35 \%)$ & \\
\hline $11-15$ years & $7(17.5 \%)$ & \\
\hline$>15$ years & $9(22.5 \%)$ & \\
\hline Type of MS & & N/A \\
\hline Relapsing-remitting (RRMS) & $25(62.5 \%)$ & \\
\hline Secondary progressive (SPMS) & $13(32.5 \%)$ & \\
\hline Primary progressive (PPMS) & $2(5 \%)$ & \\
\hline Depression (BDI) & & N/A \\
\hline Overall mean (SD) & $10( \pm 6.0)$ & \\
\hline Minimal (0-9) & $23(57.5 \%)$ & \\
\hline Mild (10-19) & $12(30 \%)$ & \\
\hline Moderate (20-29) & $5(12.5 \%)$ & \\
\hline Severe $(\geq 30)$ & $0(0 \%)$ & \\
\hline Fatigue Scale for Motor and Cognition (FSMC) & & N/A \\
\hline Overall mean (SD) & $70( \pm 16.7)$ & \\
\hline No fatigue $(20-42)$ & $3(7.5 \%)$ & \\
\hline Mild fatigue (43-52) & $1(2.5 \%)$ & \\
\hline Moderate fatigue (53-62) & $5(12.5 \%)$ & \\
\hline Severe fatigue $(\geq 63)$ & $31(77.5 \%)$ & \\
\hline Gait function $^{\mathrm{a}}$ & & N/A \\
\hline Not impaired & $32(80 \%)$ & \\
\hline Moderately impaired & $5(12.5 \%)$ & \\
\hline Severely impaired & $2(5 \%)$ & \\
\hline Missing data & $1(2.5 \%)$ & \\
\hline Manual function ${ }^{\mathrm{a}}$ & & N/A \\
\hline Not impaired & $36(90 \%)$ & \\
\hline Moderately impaired & $3(7.5 \%)$ & \\
\hline Severely impaired & $0(0 \%)$ & \\
\hline Missing data & $1(2.5 \%)$ & \\
\hline
\end{tabular}

SD: standard deviation; EDSS: Expanded Disability Status Scale; MS: multiple sclerosis; BDI: Becks Depression Inventory; FSMC: Fatigue Scale of Motor and Cognition.

aData gathered post hoc from medical files.

Functional MRI. Six functional MRI of 4.53-minute sessions with echo planar imaging (EPI) sequences were acquired: 36 slices per volume, voxel resolution: $2 \mathrm{~mm} \times 2 \mathrm{~mm} \times 3 \mathrm{~mm}$, interleaved slice order: field of 
vision $(\mathrm{FOV})=192 \mathrm{~mm}$, flip angle $=80^{\circ}, \mathrm{TR}=2.5$ seconds, $\mathrm{TE}=30 \mathrm{~ms}$ while participants performed in the $N$-back task.

$N$-back task. Visual stimuli were displayed at the center of a screen as white color letters on a gray background (Presentation ${ }^{\circledR}$ 16.4, www.neurobs.com). The screen was viewed via a mirror above the head coil. The size of the letters was $2.4^{\circ}(\mathrm{h}) \times 2.4^{\circ}(\mathrm{w})$ of visual angle.

The $N$-back task had two difficulty levels $(N-1$ and $N-2$ back). Each session consisted of 8 randomized task blocks (33 seconds each, $4 \times N-1$ back and $4 \times N$ -2 back) containing a cue phase, displayed for 3 seconds, indicating the task $(N-1$ or $N-2)$ to be completed, followed by 15 successive letter stimuli. Letters from A to L (excluding the letter "I" due to the similarity with "J") were presented for $500 \mathrm{~ms}$ each, with an inter stimulus interval (ISI) randomly distributed between 1 and 7 seconds following a gamma distribution to enhance the efficiency for event-related fMRI. ${ }^{21}$ Participants performed a speeded buttonpress with the right index finger when the presented letter was identical to the previously shown $(N-1$ back) or to the letter presented two trials before $(N-2$ back). Each task block contained four to five targets without immediate letter repetition in $N-2$ back blocks. $N-2$ back targets were absent in $N-1$ back blocks to avoid confusion (see Figure 1). The experiment was performed in six sessions of 4.53 minutes each. For each task, RTs were calculated only from correct responses and were averaged per session. The first two and the last two sessions were combined to produce values for the beginning (beg) and end. Similarly, the hit ratio (HR) was calculated by dividing the number of hits by the number of targets for each task and each session. HRs were then also combined for the first two (begin) and last two (end) sessions.

Participants rated their momentary fatigue using a visual analog scale (VAS) from 1 to 10 before and after each session in the MRI, where (1) indicated extreme fatigue. VAS scores were initially recorded before and after each session resulting in seven scores, however, for the statistical analysis, only the baseline score and the final score following the last session were used for analysis, yielding two time points.

\section{Analysis}

Statistical analyses were performed using IBM SPSS Statistics software (Version 22.0, IBM Corp., Armonk,
NY). First, data were tested for normal distribution. Analyses of variance (ANOVAs) were performed on the RTs of the TAP and those obtained during the $\mathrm{N}$-back task as well as on the subjective fatigue scores obtained using the VAS. Non-normally distributed data were analyzed using non-parametric tests (Wilcoxon test for within-group and Kruskal-Wallis test for between-group comparisons). Bonferroni correction was applied when needed. Correlations were calculated using Pearson's $R$ for parametric and Spearman's Rho for non-parametric data.

MRI. MRI data were analyzed using statistical parametric mapping software (SPM8, http://www.fil.ion. ucl.ac.uk/spm/software). All scans were resliced, realigned, normalized, and smoothed using a Gaussian isotropic kernel with a full-width at half-minimum (FWHM) of $8 \mathrm{~mm}$. After high-pass filtering (1/128 seconds), movement parameters (six dimensions) from realignment were included as covariates into the model. A flexible factorial design with three factors (subjects, group, and condition) was employed for second-level analysis. No threshold was entered for the creation of the model, and the significance thresholds of the various contrasts are mentioned in the results. Sphericity assumption violations were not assumed. All contrasts were calculated using $t$-tests.

The dynamic changes during the course of the experiment were explored by contrasting trials with correct responses from the first two sessions of the $N-2$ back task with those of the last two sessions of the $N-2$ back task across groups. Small volume correction (SVC) was applied in areas known to sub-serve attention and working memory using anatomical maps (anatomy toolbox version 2.2b of SPM8). These areas included the cingulated cortex (including the anterior cingulated cortex), lingual and fusiform gyrus, cuneus and precuneus, superior parietal lobule (SPL), as well as structures of the BG. ${ }^{12}$ To further investigate effortindependent effects within the patient group in relation to fatigue, we performed multiple regression analyses with the FSMC and FSMC-cognitive domain values as covariates.

\section{Results}

\section{Fatigue measures}

Correlations. The FSMC score and the scores of its subscales, motor (FSMC-mot) and cognitive (FSMC$\operatorname{cog}$ ) fatigue, were highly correlated (FSMC-mot: $r=$ 0.93, $p<0.000$; FSMC-cog: $r=0.93, p<0.000$ ). Similarly, FSMC-mot and FSMC-cog were also correlated $(r=0.73, p<0.000)$. Notably, the FSMC 


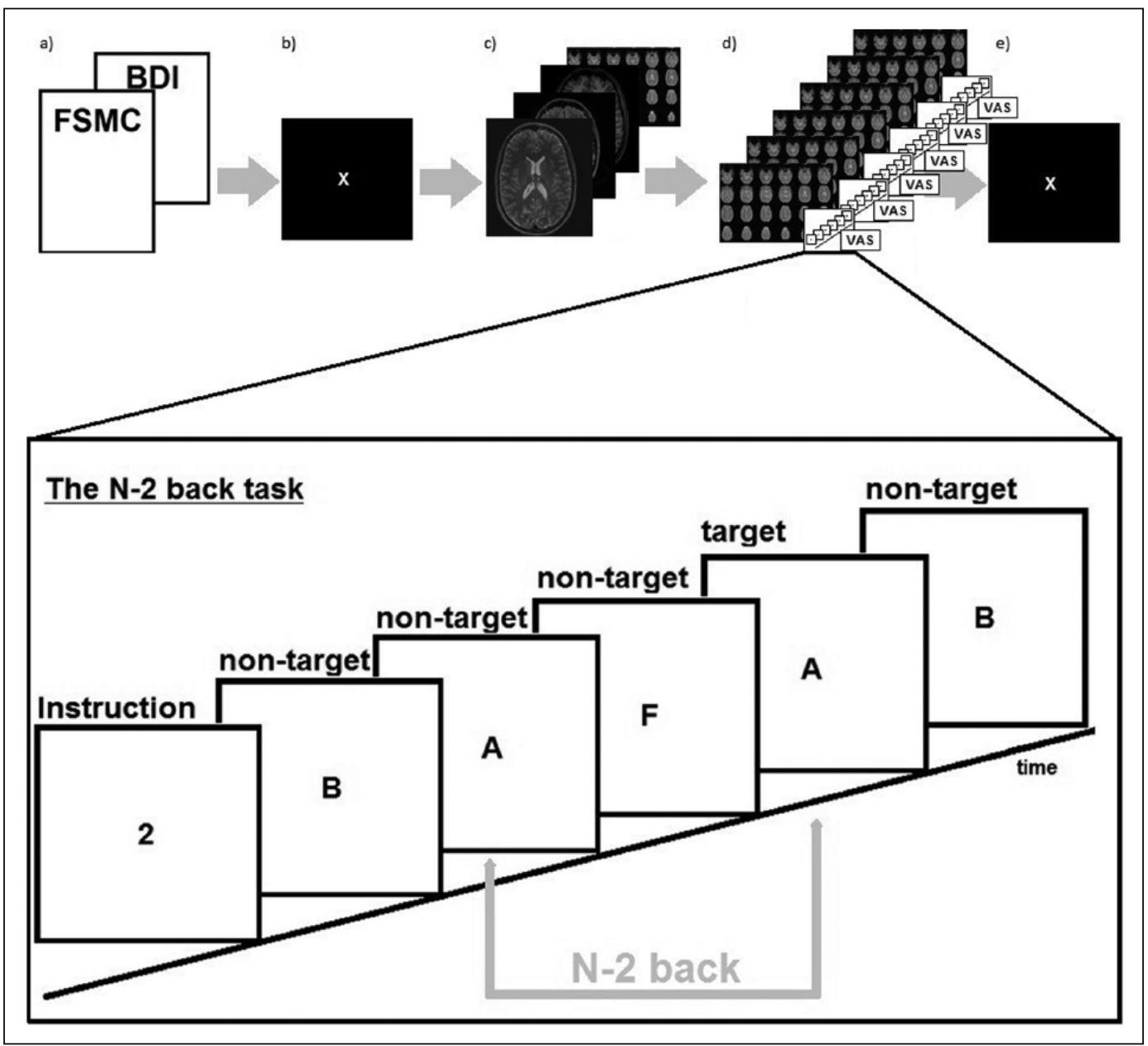

Figure 1. Study design: (a) patients completed the Fatigue Scale of Motor and Cognition (FSMC) and Beck Depression Inventory (BDI) questionnaires, (b) all participants completed the Test-battery of Attentional Performance (TAP) alertness task, (c) structural scans, and (d) functional magnetic resonance imaging (fMRI) scans while performing $N$-back tasks; in the $N-2$ task, letter stimuli were presented successively following an instruction as to which task was to be performed. Subjects were requested to press a button if the letter currently shown matched the letter shown two before it. In this example, the second presentation of the letter "A" required a response. Before and after each session, participants were requested to rate their cognitive fatigue on a Visual Analog Scale (VAS). (e) Second session TAP alertness test.

scored correlated with the BDI scores $(r=0.57, p<$ $0.000)$. The FSMC scores and the subjective fatigue (VAS) scores neither correlated with the TAP RTs nor with RTs or HRs in the $N$-back tasks.

\section{Behavioral measures}

TAP alertness. In the alertness task, the patients responded slower and with greater variance than controls, who reacted with a mean RT of $240.4 \mathrm{~ms}( \pm 34.9$ $\mathrm{ms})$ before and $241.6 \mathrm{~ms}( \pm 35.4 \mathrm{~ms})$ following the $N$-back task in the scanner, while patients performed with a mean RT of $284.2 \mathrm{~ms}( \pm 79.8 \mathrm{~ms})$ before and $312.3 \mathrm{~ms}( \pm 80.5 \mathrm{~ms})$ after the $N$-back task in the scanner. RTs were analyzed using a $2 \times 2$ ANOVA with the factors group and time point. The results indicated a main effect of group $(F(1,60)=12.731, p=0.001)$ in the absence of an interaction between the factors $(F(1$, $60)=2.662, p=0.108)$. The factor time point, however, showed a trend toward significance $(F(1,60)=$ $3.165, p=0.08)$. This trend was analyzed using a repeated-measures ANOVA (rANOVA) with the factor TAP time point (T1 and T2). Results showed a significant increase in RTs in the patient group $(F(1,39)$ $=5.53, p<0.05)$, but not in the control group $(F(1$, 21) $=0.08, p=0.78$ )

Fatigue measurements. A rANOVA with the factors group and time point revealed a main effect for each factor (group $(F(1,60)=6.54, p=0.013$ ); time point $(F(1,60)=44, p<0.000))$ and a group by time point interaction $(F(1,60)=5.924, p=0.018)($ see Figure 2$)$. 


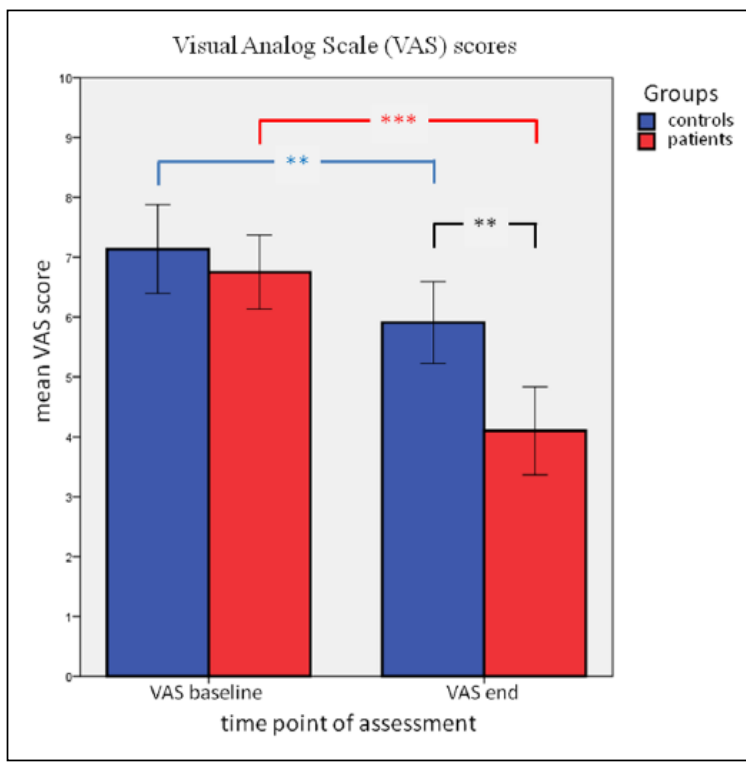

Figure 2. Mean visual analog scale (VAS) scores of fatigue for patients (in red) and controls (in blue) for the first measurement (VAS begin) and the final measurement (VAS end). Low values indicate high fatigue. Also indicated are the significant differences in both between and within groups.

${ }^{*} p<0.05 ; * * p<0.005 ; * * * p<0.001$.

The interaction was due to the fact that patients reported significantly greater subjective feelings of fatigue than controls at the end of the experiment $(F(1,60)=10.91, p=0.002)$ indicating effort-related fatigue. The groups did not differ in their baseline evaluation of subjective fatigue.

\section{$N$-back task performance}

Accuracy. In general, patients achieved a mean HR of $0.92( \pm 0.08)$ and $0.79( \pm 0.14)$ for the $N-1$ and $N-2$ task, respectively, while controls achieved a mean HR of $0.96( \pm 0.02)$ and $0.87( \pm 0.11)$ for the $N-$ 1 and $N-2$ task, respectively. The HRs for each task at the beginning and at the end of the $N$-back tasks are shown in Table 2.

As the HRs for the $N-1$ task were non-normally distributed, non-parametric tests were used for the analyses. Controls performed with a higher accuracy in the $N-1\left(\chi^{2}(1)=10.68, p<0.01\right)$ and the $N-2$ task $\left(\chi^{2}(1)=7.53, p<0.01\right)$. Both groups exhibited a practice effect in the $N-2$ task (controls: $Z=3.323, p<$ $0.000, n=22$; patients: $Z=3.441, p<0.000, n=40$ ), while patients dropped in accuracy in the $N-1$ task $(Z=-1.984, p<0.05, n=40)$.

$R T$. The RTs of the patients were over all higher than those of controls (see Table 2). The RT data were normally distributed and were submitted to an ANOVA with the factors group (patients and controls), task ( $N-1$ back and $N-2$ back), and time point (begin and end). Main effects were observed for the factors group $(F(1,1)=42.68, p<0.001)$, task $(F(1$, $1)=68.76, p<0.001)$, and a strong trend for the factor time point $(F(1,1)=3.84, p=0.051)$. A significant interaction was found between the factors task and time point $(F(1,1)=5.30, p<0.05)$ that was caused by a practice effect in the $N-2$ task (rANOVA: controls: $F(1,21)=13.52, p=0.001$; patients: $F(1,39)=$ $13.77, p=0.001)$. No significant effects were found for the $N-1$ task RTs.

\section{Functional MRI}

Group differences. To investigate group differences, the hemodynamic activity elicited by the $N-1$ back and the $N-2$ back tasks was each contrasted between controls and patients, revealing a decrease in activity within the left fusiform gyrus in the patient group compared to the control group for the $N-1$ task. However, increased activations for the patient group in the left motor and somatosensory areas, as well as the left supramarginal gyrus were also observed (Figure 3(a)). For the $N-2$ task, increased activity in patients was also observed in the same motor regions as above, with the addition of increased activity in the right supplementary motor area (SMA), the right supramarginal gyrus and the right insular lobe. Furthermore, reduced activity in the left caudate nucleus was evident for the $N-2$ task in the patient group (Figure 3(b)).

Effort-dependent changes of brain activity. To compare the neural activations at the beginning of the experiment with activations toward the end of the experiment, data from the first two sessions were contrasted versus those from the last two sessions during the more difficult $N-2$ task since behavioral and hemodynamic differences were more pronounced. This contrast revealed activity decreases in the left anterior insula, bilateral fusiform gyri, left precuneus, left SPL, right SMA, right middle cingulated cortex, right caudate, right pallidum, right putamen, and right amygdala in the patient group (Figure 4(a)).

Effort-independent fatigue-related brain activity changes. Patients' $N-2$ data of the first two sessions (in which effort-related changes are if at all rather small) were analyzed using a one sample $t$-test with covariate of interest of the FSMC-cognition scale scores and revealed activity in the bilateral ACC, the right middle cingulum cortex, and left paracentral lobule to positively correlate with the fatigue scores (Figure 4(b)). 
Table 2. Average reaction times (RTs) and standard deviations, as well as median hit ratios (HRs) and range for the first two (begin) and the last two (end) sessions for the $N-1$ back and $N-2$ back task for controls and patients, as well as results from between group comparisons.

\begin{tabular}{|c|c|c|c|c|}
\hline & Patients $(N=40)$ & Controls $(N=22)$ & Between group comparison ${ }^{a}$ & $p$-Value \\
\hline \multicolumn{5}{|l|}{ Task: $N-1$} \\
\hline Reaction times (ms) & Mean (SD) & & & \\
\hline Begin & $619.93( \pm 119.19)$ & $535.18( \pm 99.84)$ & $F(1,60)=8.01$ & 0.006 \\
\hline End & $636.95( \pm 104.27)$ & $529.5( \pm 71.84)$ & $F(1,60)=18.47$ & 0.000 \\
\hline Hit ratios (hits/misses) & Median (range) & & & \\
\hline Begin & $0.97(0.53-1.0)$ & $1.0(0.79-1.0)$ & $\chi^{2}=3.56$ & 0.059 \\
\hline End & $0.97(0.42-1.0)$ & $1.0(0.85-1.0)$ & $\chi^{2}=10.95$ & 0.001 \\
\hline \multicolumn{5}{|l|}{ Task: $N-2$} \\
\hline Reaction times (ms) & Mean (SD) & & & \\
\hline Begin & $789.6( \pm 117.45)$ & $710.00( \pm 126.70)$ & $F(1,60)=6.17$ & 0.016 \\
\hline End & $720.5( \pm 131.07)$ & $609.09( \pm 116.09)$ & $F(1,60)=11.09$ & 0.001 \\
\hline Hit ratios (hits/misses) & Median (range) & & & \\
\hline Begin & $0.79(0.22-1.0)$ & $0.86(0.36-1.0)$ & $\chi^{2}=3.62$ & 0.057 \\
\hline End & $0.89(0.38-1.0)$ & $0.95(0.51-1.0)$ & $\chi^{2}=4.88$ & 0.027 \\
\hline
\end{tabular}

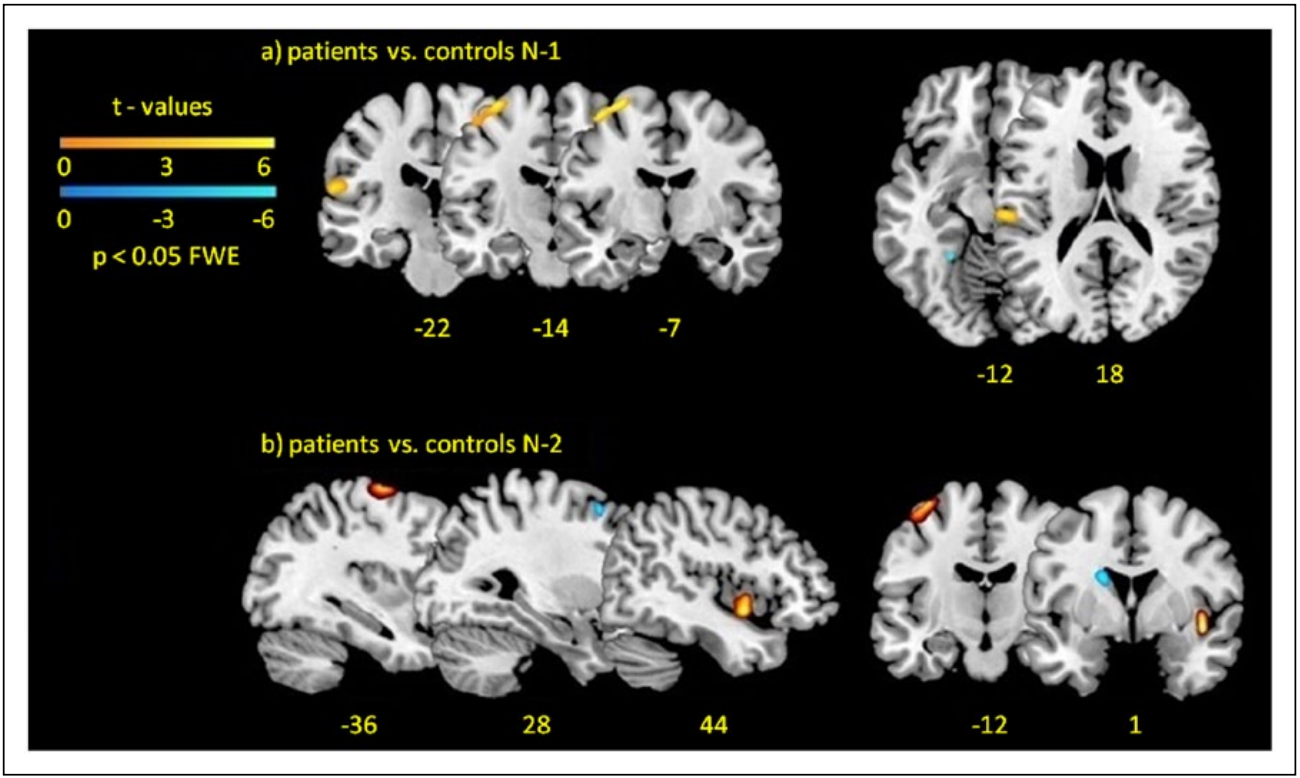

Figure 3. (a) Group differences for the $N-1$ task* $^{*}$ : greater activations in red/yellow in motor areas and left supramarginal gyrus in patients compared to controls for the $N-1$ task, less activation in patients (blue) compared to controls in the left fusiform gyrus. (b) Group differences for the $N-2$ task*: greater activations in red/yellow in motor areas and right insula in patients compared to controls for the $N-2$ task, less activation in patients (blue) compared to controls in the left caudate nucleus and right middle frontal gyrus (rMFG).

The numbers below the images indicate the axial, coronal, and sagittal coordinates in MNI space, respectively. *Please refer to section "Materials and methods."

Depression and related brain activity. Patients' $N-2$ data of the first two sessions were analyzed as a function of the BDI scores. The areas which showed correlating neural activity much resembled those correlating with fatigue. These were the right precentral gyrus, the left anterior and right middle cingulate cortex, as well as the right frontal gyrus. 


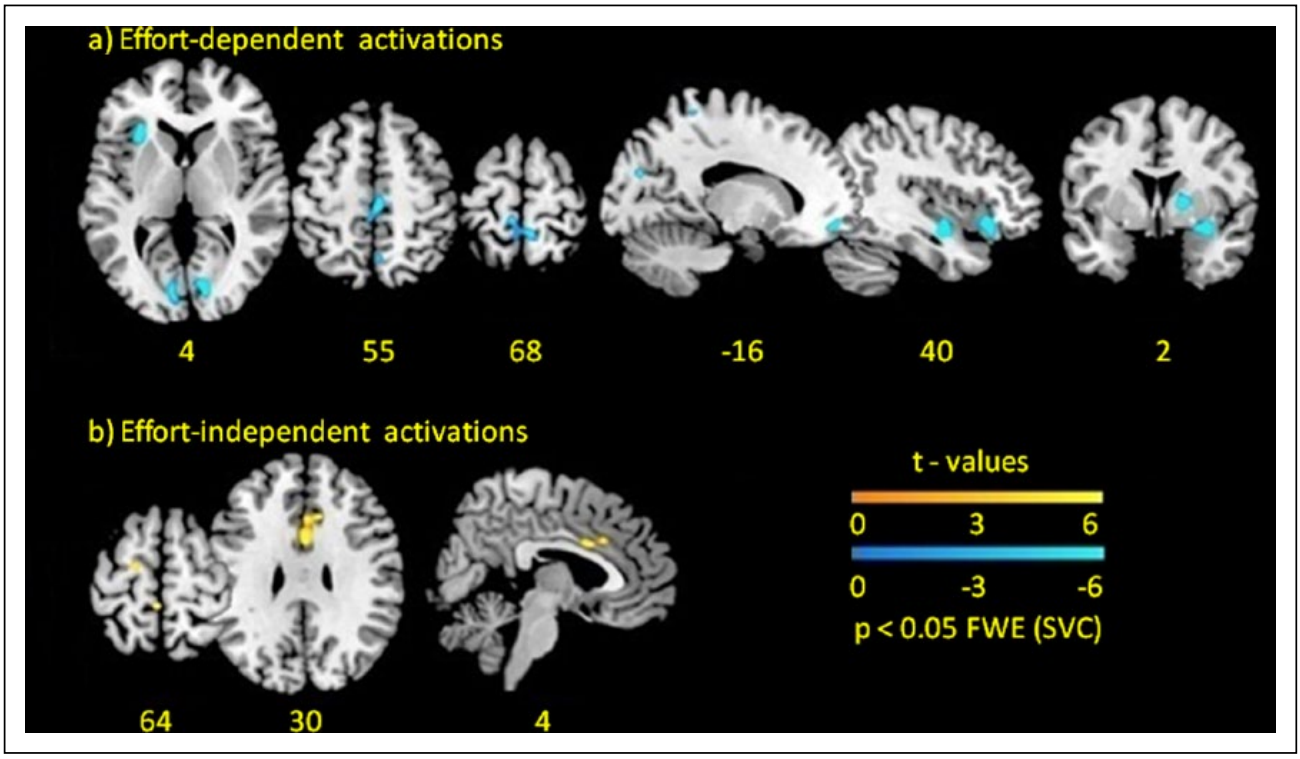

Figure 4. (a) Effort-dependent correlates: decrease in activation (displayed in blue) in the patient group as opposed to the control group at the end of the experiment versus at the beginning.* (b) Effort-independent correlates: activation (in yellow) within the bilateral anterior cingulum cortex (ACC) and left paracentral lobule.*

*All activations displayed are small volume corrected (SVC). The numbers below the images indicate the axial, coronal, and sagittal coordinates in MNI space, respectively.

\section{Discussion}

This study combined behavioral and fMRI measures to investigate the neural correlates of fatigue in patients with MS and found a generally lower performance level in patients compared to controls, further decreasing in specific tasks as a function of task length in patients but not in controls. In parallel, subjective assessments of fatigue showed a higher selfperceived fatigue increasing with task length in patients compared to controls.

Performing the task in the scanner prolonged the TAP RTs in patients but not in controls, possibly indicating an effort-dependent decrease of patient's alertness level, however, a significant interaction effect was not observed in the statistical analysis. The behavioral data of the $N$-back task showed that patients' RTs were generally slower than those of controls. Controls performed faster and more accurate in the $N-1$ task, and in the $N-2$ task both groups improved. There was a difference between patients and controls in performance and alertness already before performing in the $N$-back task, presumably reflecting the limited neural resources or cognitive fatigue in MS. ${ }^{22}$ Performance differences in patients but not in controls at the end versus beginning of the $N-1$ task were observed in the $N$-back task itself and insinuated in the alertness task performed thereafter possibly reflecting fatigue. All other behavioral measures showed a practice effect due to successive measurements. The subjective fatigue (VAS) also increased after the task exclusively in patients. In short, the behavioral data clearly indicate a baseline difference in alertness, an effortdependent performance change, and increase of subjective fatigue in patients and to a significantly lesser degree in controls.

Consistent with previous studies, ${ }^{23}$ the $N$-back task elicited activity in areas involved in attention and working memory. Activity in motor and somatosensory areas, the right supramarginal gyrus, and right insula, particularly for the more difficult $N-2$ back task, was higher in patients than in controls. Importantly, the left fusiform gyrus and the left caudate nucleus showed less activity in patients. The activity increase in the motor- and somatosensoryrelated areas in MS patients likely reflects higher activity requirements for the same motor action (e.g. pressing the response key) due to cortical and white matter damage. ${ }^{10,24,25}$ The insular cortex is involved in both interoceptive awareness and homeostasis, is proposed to be a key region for sustaining and redirecting attention, and is part of the salience network. ${ }^{26,27}$ The insula is required for verbal working memory, specifically for short-term memory of letters and selective attention. ${ }^{28}$ It is a junction point between selective attention and arousal systems and the BG recruited during complex cognitive tasks. ${ }^{29}$ The present findings add to the evidence that patients with MS display 
altered patterns of neural activity during tasks placing demands on information processing, memory, and sustained attention, ${ }^{30}$ and provide strong support for the fatigue model proposed by Chaudhuri and Behan, 4,31 who propose an involvement of the non-motor functions of the $\mathrm{BG}$ in the occurrence of fatigue in $\mathrm{MS}$.

We observed different neural correlates associated with effort-independent and effort-dependent performance changes. The effort-independent changes were analyzed in dependence of the fatigue scores (FSMCcognitive subscale). Only fMRI data from the first two sessions were included to minimize the influence of effort-dependent fatigue. High fatigue scores were associated with higher activity in the ACC (see Figure 4(b)), a region involved in cognitive functions, including error detection, performance monitoring, response selection, and attention control. ${ }^{32}$ It plays a role during the maintenance of goal-directed behavior, working memory, and inhibition and acts top-down on subconscious automatic information processing during cognitively challenging conditions involving conflict. ${ }^{33,34}$ It has previously been proposed that the ACC might be overactive in patients with cognitive fatigue $^{11}$ and MS. ${ }^{35}$ The current results support the idea of a close relationship between ACC activity and effort-independent fatigue in MS patients. The right middle cingulum also showed increased activity with higher fatigue scores. This area is involved in cognitive control, and its microstructure predicts performance breakdown in several neurodegenerative diseases. ${ }^{36}$ Another region that showed increased activity with higher fatigue scores was the left paracentral lobule, a region involved in sensory-motor processing as well as in the regulation of physiologic functions such as micturition, which are also often disturbed in MS. ${ }^{37}$ Similar regions were, however, also found to correlate with the depression scores attained from the BDI. These included the right precentral gyrus, the left anterior and right middle cingulate cortex, as well as the right frontal gyrus. Since effort-independent fatigue and depression share many symptoms, ${ }^{38,39}$ they might also share some neural correlates, which would be in line with findings in the literature. ${ }^{40}$

The effort-independent fatigue of MS patients most likely corresponds to what has been termed the "trait" ${ }^{-10}$ or general fatigue component. 5 Extending previous findings suggesting a striatal-thalamic-frontal cortical system to underlie the trait component of fatigue, this study adds frontal attention control and sensory-motor regions to the previously suggested network, thereby proving strong support to the model proposed by Chaudhuri and Behan. ${ }^{4,31}$
The second fatigue-related component refers to the effort-dependent dynamic change of fatigue in MS patients, which has been termed "state fatigue" or "fatigability." $5,8,10$ Consistently, the behavioral data indicated that this component was predominantly present in MS patients. Its neural correlates were reflected by activity decreases in the right SMA, the left anterior insula, the bilateral fusiform gyri, left precuneus, left SPL, right middle cingular cortex, right caudate nucleus, pallidum, putamen, and amygdala observed particularly in the more challenging $N-2$ back taskcondition. The anterior insula and the cingulum are part of the saliency network that plays an important role in task-level control and focal attention, ${ }^{41}$ which are core components of the task of this study. The parietal areas and the fusiform gyri are all heavily involved in visual attention and working memory. ${ }^{12}$ The pallidum and the putamen are also involved in attentional orienting, ${ }^{42}$ while the amygdala was shown to influence attention. ${ }^{43}$ In sum, the subjective increase of fatigue, paralleled by the RT increase in the alertness task, was associated with a drop of hemodynamic activity in attention-related networks in MS patients suggesting that dynamic reductions of activity in salience and attention-related networks underlie the state or fatigability component of fatigue in MS.

\section{Acknowledgements}

C.D. and M.A.S. equally contributed to the manuscript.

\section{Declaration of Conflicting Interests}

The author(s) declared no potential conflicts of interest with respect to the research, authorship, and/or publication of this article.

\section{Funding}

The author(s) disclosed receipt of the following financial support for the research, authorship, and/or publication of this article: The study was funded by the Stiftung Schmieder für Wissenschaft und Forschung, Allensbach.

\section{References}

1. Rao SM, Leo GJ, Bernardin L, et al. Cognitive dysfunction in multiple sclerosis. I. Frequency, patterns, and prediction. Neurology 1991; 41: 685-691.

2. Fisk JD, Pontefract A, Ritvo PG, et al. The impact of fatigue on patients with multiple sclerosis. Can J Neurol Sci 1994; 21: 9-14.

3. Krupp LB, LaRocca NG, Muir-Nash J, et al. The fatigue severity scale: Application to patients with 
multiple sclerosis and systemic lupus erythematosus. Arch Neurol 1989; 46: 1121-1123.

4. Chaudhuri A and Behan PO. Fatigue and basal ganglia. J Neurol Sci 2000; 179: 34-42.

5. Kluger BM, Krupp LB and Enoka RM. Fatigue and fatigability in neurologic illnesses proposal for a unified taxonomy. Neurology 2013; 80: 409-416.

6. Harrison AM, das Nair R and Moss-Morris R. Operationalising cognitive fatigability in multiple sclerosis: A Gordian knot that can be cut? Mult Scler 2017; 23: 1682-1696.

7. Tedeschi G, Dinacci D, Lavorgna L, et al. Correlation between fatigue and brain atrophy and lesion load in multiple sclerosis patients independent of disability. J Neurol Sci 2007; 263: 15-19.

8. Calabrese M, Rinaldi F, Grossi P, et al. Basal ganglia and frontal/parietal cortical atrophy is associated with fatigue in relapsing-remitting multiple sclerosis. Mult Scler 2010; 16: 1220-1228.

9. Sepulcre J, Masdeu JC, Goni J, et al. Fatigue in multiple sclerosis is associated with the disruption of frontal and parietal pathways. Mult Scler 2009; 15: 337-344.

10. Genova HM, Rajagopalan V, Deluca J, et al. Examination of cognitive fatigue in multiple sclerosis using functional magnetic resonance imaging and diffusion tensor imaging. PLoS ONE 2013; 8: e78811.

11. Dobryakova E, DeLuca J, Genova HM, et al. Neural correlates of cognitive fatigue: Corticostriatal circuitry and effort-reward imbalance. J Int Neuropsychol Soc 2013; 19: 849-853.

12. Stoppel CM, Boehler CN, Strumpf H, et al. Distinct representations of attentional control during voluntary and stimulus-driven shifts across objects and locations. Cereb Cortex 2013; 23: 1351-1361.

13. Merkel C, Hopf JM, Heinze HJ, et al. Neural correlates of multiple object tracking strategies. Neuroimage 2015; 118: 63-73.

14. Hanken K, Eling $P$ and Hildebrandt $H$. Is there a cognitive signature for MS-related fatigue? Mult Scler 2015; 21: 376-381.

15. Penner I, Raselli C, Stöcklin M, et al. The Fatigue Scale for Motor and Cognitive functions (FSMC): Validation of a new instrument to assess multiple sclerosis-related fatigue. Mult Scler 2009; 15: 1509-1517.

16. Hautzinger M, Bailer M, Worall H, et al. Beck Depression Inventory (BDI). Bern: Huber, 1994.

17. Zimmermann P and Fimm B. A test battery for attentional performance. Appl Neuropsychol Attention 2002; 20: 110-151.
18. Claros-Salinas D, Dittmer N, Neumann M, et al. Induction of cognitive fatigue in MS patients through cognitive and physical load. Neuropsychol Rehabil 2013; 23: 182-201.

19. Neumann M, Sterr A, Claros-Salinas D, et al. Modulation of alertness by sustained cognitive demand in MS as surrogate measure of fatigue and fatigability. J Neurol Sci 2014; 340: 178-182.

20. Weinges-Evers N, Brandt AU, Bock M, et al. Correlation of self-assessed fatigue and alertness in multiple sclerosis. Mult Scler 2010; 16: 1134-1140.

21. Friston KJ, Zarahn E, Josephs O, et al. Stochastic designs in event-related fMRI. Neuroimage 1999; 10: 607-619.

22. Sailer M, Fischl B, Salat D, et al. Focal thinning of the cerebral cortex in multiple sclerosis. Brain 2003; 126: 1734-1744.

23. Nee DE, Brown JW, Askren MK, et al. A metaanalysis of executive components of working memory. Cereb Cortex 2013; 23: 264-282.

24. Buckle GJ. Functional magnetic resonance imaging and multiple sclerosis: The evidence for neuronal plasticity. J Neuroimag 2005; 15: 82S-93S.

25. Filippi M and Rocca MA. Disturbed function and plasticity in multiple sclerosis as gleaned from functional magnetic resonance imaging. Curr Opin Neurol 2003; 16: 275-282.

26. Cauda F, Costa T, Torta DM, et al. Meta-analytic clustering of the insular cortex: Characterizing the meta-analytic connectivity of the insula when involved in active tasks. Neuroimage 2012; 62: 343-355.

27. Gasquoine PG. Contributions of the insula to cognition and emotion. Neuropsychol Rev 2014; 24: 77-87.

28. Augustine JR. Circuitry and functional aspects of the insular lobe in primates including humans. Brain Res Rev 1996; 22: 229-244.

29. Eckert MA, Menon V, Walczak A, et al. At the heart of the ventral attention system: The right anterior insula. Hum Brain Mapp 2009; 30: 2530-2541.

30. Staffen W, Mair A, Zauner H, et al. Cognitive function and $\mathrm{FMRI}$ in patients with multiple sclerosis: Evidence for compensatory cortical activation during an attention task. Brain 2002; 125: 1275-1282.

31. Chaudhuri A and Behan PO. Fatigue in neurological disorders. Lancet 2004; 363: 978-988.

32. Walton ME, Bannerman DM, Alterescu K, et al. Functional specialization within medial frontal cortex of the anterior cingulate for evaluating effort-related decisions. J Neurosci 2003; 23: 6475-6479.

33. Gasquoine PG. Localization of function in anterior cingulate cortex: From psychosurgery to functional 
Visit SAGE journals online journals.sagepub.com/ home/msj

@ SAGE journals neuroimaging. Neurosci Biobehav Rev 2013; 37 : 340-348.

34. Wendelken C, Ditterich J, Bunge SA, et al. Stimulus and response conflict processing during perceptual decision making. Cogn Affect Behav Neurosci 2009; 9: 434-447.

35. Roelcke U, Kappos L, Lechner-Scott J, et al. Reduced glucose metabolism in the frontal cortex and basal ganglia of multiple sclerosis patients with fatigue: A 18F-fluorodeoxyglucose positron emission tomography study. Neurology 1997; 48: $1566-1571$.

36. Metzler-Baddeley C, Jones DK, Steventon J, et al. Cingulum microstructure predicts cognitive control in older age and mild cognitive impairment. J Neurosci 2012; 32: 17612-17619.

37. MacKenzie-Graham A, Kurth F, Itoh Y, et al. Disability-specific atlases of gray matter loss in relapsing-remitting multiple sclerosis. JAMA Neurol 2016; 73: 944-953.
38. Lobentanz I, Asenbaum S, Vass K, et al. Factors influencing quality of life in multiple sclerosis patients: Disability, depressive mood, fatigue and sleep quality. Acta Neurol Scand 2004; 110: 6-13.

39. Kroencke DC, Lynch SG and Denney DR. Fatigue in multiple sclerosis: Relationship to depression, disability, and disease pattern. Mult Scler J 2000; 6: 131-136.

40. Gobbi C, Rocca MA, Riccitelli G, et al. Influence of the topography of brain damage on depression and fatigue in patients with multiple sclerosis. Mult Scler 2014; 20: 192-201.

41. Nelson SM, Dosenbach NU, Cohen AL, et al. Role of the anterior insula in task-level control and focal attention. Brain Struct Funct 2010; 214: 669-680.

42. Hassler R. Striatal regulation of adverting and attention directing induced by pallidal stimulation. Appl Neurophysiol 1979; 42: 98-102.

43. Peck CJ and Salzman CD. The amygdala and basal forebrain as a pathway for motivationally guided attention. J Neurosci 2014; 34: 13757-13767. 